\title{
Complications with oxycodone and naloxone
}

\author{
Alicia Ward \\ Consultant \\ Michaela del Campo \\ Pharmacist \\ Kathy Hauser \\ Consultant \\ Central Adelaide Palliative \\ Care Services \\ Adelaide
}

Aust Prescr 2017:40:156-7 https://doi.org/10.18773/ austprescr.2017.018

\section{Case 1}

A 43-year-old woman with metastatic breast cancer was admitted with constipation. She had liver metastases, ascites and impaired synthetic function (Child-Pugh B),' but aspartate aminotransferase and alanine aminotransferase were within normal limits.

Her analgesia was changed from sustained-release morphine to a combination product of prolongedrelease oxycodone with naloxone. Within two hours of the first dose she developed severe pain. This was uncontrolled by morphine and ketamine infusion.

The pain persisted for 12 hours after receiving the prolonged-release oxycodone with naloxone. The patient was recommenced on her previous morphine regimen and achieved ongoing stable pain control.

\section{Case 2}

A 50-year-old man with metastatic prostate cancer had increasing pain despite increasing doses of prolonged-release oxycodone with naloxone (up to $40 / 20$ mg twice daily) plus controlledrelease oxycodone (10 $\mathrm{mg}$ twice daily). The man reported no effect from using immediate-release oxycodone for breakthrough pain. He had liver metastases but aspartate aminotransferase and alanine aminotransferase were within normal limits (Child-Pugh A).

The prolonged-release oxycodone with naloxone was ceased and controlled-release oxycodone $20 \mathrm{mg}$ twice daily was commenced (a significantly lower opioid dose). Within two days, the patient's pain and functional status greatly improved enabling his cancer therapy to be resumed.

\section{Case 3}

A 51-year-old man with a sacral chondrosarcoma and poorly controlled pain became narcotised (lifethreatening overdose) when prolonged-release oxycodone with naloxone was ceased while on a stable dose of methadone. Once the methadone was cleared over the next few days his analgesia was significantly improved on a comparatively lower dose morphine infusion (without the multiple adjuvant analgesics previously required). He had no liver metastases, normal aspartate aminotransferase and alanine aminotransferase (Child-Pugh A) with fatty liver on ultrasound.

\section{Comment}

The combination of prolonged-release oxycodone with naloxone is marketed to reduce pain and opioidinduced constipation. Naloxone aims to counteract the effect of oxycodone on the gut over 12 hours as it antagonises opioid receptors in the gastrointestinal tract. As naloxone then undergoes extensive first-pass metabolism in the liver, insignificant amounts enter the systemic circulation. In healthy people its bioavailability is less than $3 \%$. The combination is contraindicated in moderate-severe liver dysfunction (Child-Pugh B-C).

We have observed prescription of higher than recommended doses and the commencement or continuation of prolonged-release oxycodone with naloxone despite deteriorating liver function. This needs to be avoided. If a patient with liver impairment is changed from oxycodone with naloxone to a single opioid formulation, start with a lower equivalent dose of the new opioid (i.e. $50 \%$ of the approximate equianalgesic dose) and monitor the patient carefully for adverse effects or toxicity. ${ }^{2}$

In cases 1 and 2 liver metastases appeared to be associated with a systemic effect of naloxone, despite normal or only mildly abnormal liver function. In case 3, the patient became narcotised due to the methadone effect after the opioid antagonist effect of naloxone had worn off. The patient's naloxone concentrations may have been higher than expected due to fatty liver disease, or through an interaction with methadone which has been shown to reduce the metabolism of naloxone. ${ }^{3}$

These and other published cases $^{4-6}$ suggest reduced first-pass metabolism results in increased concentrations of naloxone reaching the systemic circulation and antagonising the effect of opioids. This causes reduced analgesia and elevates the risk of opioid overdose when changing between opioids. The explanation could be a reduced liver capacity to metabolise naloxone.

\section{Recommendations}

We suggest:

- not using prolonged-release oxycodone with naloxone if there is any degree of liver dysfunction as there is a risk of poor analgesia or withdrawal

- avoiding prolonged-release oxycodone with naloxone in combination with other long-acting opioids, especially methadone 
- further research to understand how to safely cease or rotate the prolonged-release oxycodone with naloxone when prescribed alone or with other opioids to reduce the risk of opioid toxicity.
Conflicts of interest: none declared

4. Burns E, McWilliams K, Ross C. A cautionary tale of oral naloxone. J Pain Symptom Manage 2014;47:el-e2. https://doi.org/10.1016/j.jpainsymman.2013.10.013

5. Kang JH, Lee GW, Shin SH, Bruera E. Opioid withdrawal syndrome after treatment with low-dose extended-release oxycodone and naloxone in a gastric cancer patient with portal vein thrombosis. J Pain Symptom Manage 2013;46:e15-7. https://doi.org/10.1016/j.jpainsymman.2013.02.009

6. Mercadante S, Ferrera P, Adile C. High doses of oxycodonenaloxone combination may provide poor analgesia. Support Care Cancer 2011;19:1471-2. https://doi.org/10.1007/ s00520-011-1205-x 